\title{
UNIQUENESS OF THE CRITICAL POINT OF THE SOLUTIONS TO SOME SEMILINEAR ELLIPTIC BOUNDARY VALUE PROBLEMS IN $\mathbf{R}^{2}$
}

\author{
SHIGERU SAKAGUCHI
}

\begin{abstract}
We consider some two-dimensional semilinear elliptic boundary value problems over a bounded convex domain in $\mathbf{R}^{2}$ and show the uniqueness of the critical point of the solutions.
\end{abstract}

\section{INTRODUCTION AND RESULTS}

Uniqueness of the critical point of the solutions to elliptic boundary value problems over a convex domain has been shown by many authors.

Concerning the Dirichlet problem there are many results. In [19], Sperb considered the semilinear elliptic Dirichlet problem over a strictly convex domain $\Omega$ in $\mathbf{R}^{2}$ :

$$
\Delta u=f(u) \text { in } \Omega \text { and } u=0 \text { on } \partial \Omega,
$$

and he showed that any positive solution $u$ to this has only one critical point for some $f$. His proof is based on an idea of Payne [17].

On the other hand, many results concerning concavity properties (especially, convexity of level sets) of solutions to elliptic boundary value problems over a convex domain in $\mathbf{R}^{n}(n \geq 2)$ were obtained by various authors (see [3, 4, 9, $10,11,12,13,14]$ especially, see Kawohl [10] and its references). To illustrate their methods, we consider the Dirichlet problem

$$
\begin{cases}\Delta u=-1 & \text { in } \Omega, \\ u=0 & \text { on } \partial \Omega,\end{cases}
$$

where $\Omega$ is a bounded convex domain in $\mathbf{R}^{n}$. Put $v=-\sqrt{u}$. Then $v$ satisfies

$$
\left\{\begin{array}{l}
\Delta v=-\left(|\nabla v|^{2}+\frac{1}{2}\right) \frac{1}{v} \text { in } \Omega, \\
v=0 \text { on } \partial \Omega, \text { and } \partial v / \partial \nu=\infty \text { on } \partial \Omega,
\end{array}\right.
$$

where $\partial v / \partial \nu$ denotes the outer normal derivative of $v$ on $\partial \Omega$. Put $f(v, \nabla v)$ $=$ the right-hand side of this equation. Note that $f(\cdot, \nabla v)^{-1}$ is convex, and

Received by the editors July 28,1988 .

1980 Mathematics Subject Classification (1985 Revision). Primary 35B50; Secondary 35J25, $35 \mathrm{~J} 60$. 
$f$ together with its derivative $\partial f / \partial v$ is positive in $\Omega$. Kawohl [9] and Kennington [11] improved Korevaar's convexity maximum principle and proved the convexity of $v$. Their proofs are based on convexity maximum principles combined with the fact that $\partial v / \partial \nu=\infty$ on $\partial \Omega$. (This fact and strict convexity of $\Omega$ guarantee that $v$ is strictly convex near the boundary $\partial \Omega$.) Caffarelli and Friedman [3] showed the strict convexity of $v$, when the dimension $n$ is equal to 2. Their proof is based on an application of maximum principle to the function $\operatorname{det}\left(\partial^{2} v / \partial x_{i} \partial x_{j}\right)$ and the fact that $\partial v / \partial \nu=\infty$ on $\partial \Omega$, and the method of continuity with respect to the domain $\Omega$ (that is, they considered the continuous deformation of $\Omega$ into a ball). Furthermore, Korevaar and Lewis [14] generalized this method to $\mathbf{R}^{n} \quad(n \geq 2)$. All these methods need some algebraic structures of the equation (especially, the convexity of $f(\cdot, \nabla v)^{-1}$ ) and the fact that $\partial v / \partial \nu=\infty$ on $\partial \Omega$.

Concerning the problem which is not Dirichlet, we know the result of Chen. In [6], Chen showed that the capillary free surface over a convex domain in $\mathbf{R}^{2}$ has only one minimal point. Precisely, for a bounded convex domain $\Omega$ in $\mathbf{R}^{2}$ with boundary $\partial \Omega$, he considered the problem

$$
\left\{\begin{array}{l}
\operatorname{div} T u=2 H \quad \text { in } \Omega \\
T u \cdot \nu=\cos \gamma \quad \text { on } \partial \Omega
\end{array}\right.
$$

where $H(H>0), \gamma(0<\gamma<\pi / 2)$ are constants satisfying $2 H|\Omega|=$ $\cos \gamma|\partial \Omega|(|\Omega|$ is the area of $\Omega$ and $|\partial \Omega|$ is the length of $\partial \Omega)$ and $\nu$ denotes the unit outer normal vector to $\partial \Omega$ and $T u=\left(1+|\nabla u|^{2}\right)^{-1 / 2} \nabla u$. His results say that the solution $u$ to (1.4) has only one critical point under the hypothesis of the existence of the solution with $\gamma=0$. His proof is based on a nice comparison technique found in Chen and Huang [5] and the method of continuity with respect to $\gamma$ and the result of Chen and Huang [5] (that is, "the solution with $\gamma=0$ is strictly convex").

In this paper we consider some two-dimensional semilinear elliptic boundary value problems, which are not Dirichlet, and prove the uniqueness of the critical point of the solution. Our methods are based on an idea of Chen [6]. Precisely, let $\Omega$ be a bounded convex domain in $\mathbf{R}^{2}$ with smooth boundary $\partial \Omega$, and let $f$ be a real valued $C^{\infty}$-nondecreasing function on $\mathbf{R}$, which is positive somewhere.

Now, our results are the following:

Theorem 1. Let $u \in C^{2}(\bar{\Omega})$ be the solution to

$$
\left\{\begin{array}{l}
\Delta u=f(u) \text { in } \Omega, \\
\frac{\partial u}{\partial \nu}=c \quad \text { on } \partial \Omega,
\end{array}\right.
$$

where $c$ is a positive constant. Then $u$ has one and only one critical point in $\Omega$. 
Theorem 2. Suppose that $f(0)$ is positive. Let $u \in C^{2}(\bar{\Omega})$ be the solution to

$$
\left\{\begin{array}{l}
\Delta u=f(u) \text { in } \Omega, \\
\partial u / \partial \nu+\beta u=0 \text { on } \partial \Omega,
\end{array}\right.
$$

where $\beta$ is a positive constant. Then $u$ has one and only one critical point in $\Omega$.

Our theorems concern only qualitative properties of the solutions, so only under the hypothesis of the existence of solutions we show the uniqueness of the critical point of the solutions. For the existence of solutions, for example, see Lieberman [15] or Lieberman and Trudinger [16].

In the following sections we prove these theorems. $\S 2$ provides some preliminary results for the problems $(1.5),(1.6)$. In $\S 3$ we introduce two families of problems indexed by a bounded closed interval $[0,1]$ in order to use the method of continuity. In $\S 4$ we prove several basic lemmas with the help of one modification of Chen and Huang's comparison technique. $\S 5$ is devoted to the completion of the proofs.

\section{Preliminaries}

First of all, using the strong maximum principle, we get

Proposition 2.1. Let $u \in C^{2}(\bar{\Omega})$ be the solution to (1.5), or (1.6). Then $f(u)$ is positive in $\bar{\Omega}$ and $\partial u / \partial \nu>0$ on $\partial \Omega$. Furthermore, $u<0$ in $\bar{\Omega}$ in the case of (1.6).

Proof. Case of (1.5). Since $\partial u / \partial \nu=c>0$ on $\partial \Omega$, there exists a point $x_{0} \in \Omega$ satisfying $u\left(x_{0}\right)=\min _{\Omega} u$. Then $f\left(u\left(x_{0}\right)\right)=\Delta u\left(x_{0}\right) \geq 0$. Hence we get

$$
f(u(x)) \geq f\left(u\left(x_{0}\right)\right) \geq 0 \text { for any } x \in \bar{\Omega},
$$

since $f$ is nondecreasing. Suppose that $f\left(u\left(x_{0}\right)\right)=0$. Put $v(x)=u(x)-u\left(x_{0}\right)$. Then $v$ satisfies

$$
\left\{\begin{array}{l}
\Delta v=a(x) v \quad \text { in } \Omega, \\
v \geq 0 \text { and } a(x) \geq 0 \text { in } \Omega,
\end{array}\right.
$$

where $a(x)=\int_{0}^{1} f^{\prime}\left(u\left(x_{0}\right)+\theta\left(u(x)-u\left(x_{0}\right)\right)\right) d \theta$ (' denotes differentiation). It follows from the strong maximum principle that $v$ cannot achieve a nonpositive minimum in $\Omega$. This contradicts the fact that $v\left(x_{0}\right)=0$. Therefore

$$
f\left(u\left(x_{0}\right)\right)>0 \text {. }
$$

Case of (1.6). Since $f(0)$ is positive, $u$ satisfies

$$
\left\{\begin{array}{l}
\Delta u-a(x) u>0 \text { in } \Omega, \\
\partial u / \partial \nu+\beta u=0 \text { on } \partial \Omega \text { and } a(x) \geq 0 \text { in } \Omega,
\end{array}\right.
$$

where $a(x)=\int_{0}^{1} f^{\prime}(\theta u(x)) d \theta$. The strong maximum principle implies that $u$ cannot achieve a nonnegative maximum in $\Omega$. Suppose that $\max _{\bar{\Omega}} u \geq 0$. Then there exists a point $x_{0} \in \partial \Omega$ satisfying $u\left(x_{0}\right)=\max _{\bar{\Omega}} u \geq 0$. It follows from the strong maximum principle that $\partial u\left(x_{0}\right) / \partial \nu>0$. This contradicts the fact that 
$\partial u\left(x_{0}\right) / \partial \nu+\beta u\left(x_{0}\right)=0$. Therefore $u<0$ in $\bar{\Omega}$. Hence $\partial u / \partial \nu=-\beta u>0$ on $\partial \Omega$, and there exists a point $x_{0} \in \Omega$ satisfying $u\left(x_{0}\right)=\min _{\Omega} u$. Then, for any $x \in \bar{\Omega}$

$$
f(u(x)) \geq f\left(u\left(x_{0}\right)\right)=\Delta u\left(x_{0}\right) \geq 0 .
$$

As in the case of (1.5), using the function $v(x)=u(x)-u\left(x_{0}\right)$, we see that $f\left(u\left(x_{0}\right)\right)>0$. This completes the proof.

Concerning the uniqueness and the regularity of the solution, we have

Proposition 2.2. (1) The solution to (1.5) is unique up to an additive constant. (2) The solution to (1.6) is unique. (3) The solution to (1.5) or (1.6) belongs to $C^{\infty}(\overline{\mathbf{\Omega}})$.

Proof. Since $f$ is nondecreasing, (1) and (2) follow from the strong maximum principle. Since $f$ is smooth, the regularity theory of elliptic partial differential equations implies (3).

When $f$ is a positive constant, from the theory of linear elliptic partial differential equations (see for example Gilbarg and Trudinger [7]) we obtain

Proposition 2.3. There exists a solution $u \in C^{\infty}(\bar{\Omega})$ to the problem

$$
\Delta u=k \text { in } \Omega \text { and } \partial u / \partial \nu=c \text { on } \partial \Omega,
$$

where $k=|\partial \Omega| c /|\Omega|$ and $c$ is the positive constant in (1.5). Furthermore the solution to this problem is unique up to an additive constant.

\section{FAMILIES OF PROBLEMS FOR THE METHOD OF CONTINUITY}

For $t \quad(0 \leq t \leq 1)$, we introduce the following problems:

$$
\left\{\begin{array}{l}
\Delta u=t f(u)+(1-t) k \text { in } \Omega, \\
\partial u / \partial \nu=c \text { on } \partial \Omega,
\end{array}\right.
$$

where $k=|\partial \Omega| c /|\Omega|$ and $c$ is the positive constant in (1.5),

$$
\left\{\begin{array}{l}
\Delta u=t f(u)+(1-t) f(m) \text { in } \Omega \\
\partial u / \partial \nu+\beta u=0 \text { on } \partial \Omega
\end{array}\right.
$$

where $m$ is the minimum value of the unique solution to (1.6) and $\beta$ is the positive constant in (1.6).

Remark 3.1. $(3.1 .0)=(2.1),(3.1 .1)=(1.5),(3.2 .1)=(1.6)$.

Remark 3.2. Concerning the uniqueness of the solution to these problems, we obtain the same results as in Proposition 2.2, since the right-hand sides of these equations are nondecreasing functions with respect to $u$. Also we have the same results as in Proposition 2.1 to these problems, if we replace $f(u)$ by the right-hand sides of these equations. 
Concerning the existence, by the method of sub- and supersolutions we obtain Proposition 3.3. Under the hypothesis of the existence of the solution to the problem (1.5) we see that there exists a solution $u_{t} \in C^{\infty}(\bar{\Omega})$ to the problem (3.1.t) satisfying

$$
\left\|u_{t}\right\|_{C^{2+\alpha}(\bar{\Omega})} \leq C \text { for all } t \in[0,1],
$$

where $C$ and $\alpha$ are positive constants independent of $t \in[0,1]$, and the solution to (3.1.t) is unique up to an additive constant.

Proposition 3.4. Under the hypothesis of the existence of the solution to (1.6) we see that there exists a unique solution $u_{t} \in C^{\infty}(\bar{\Omega})$ to the problem (3.2.t) satisfying the same inequality as (3.3) for all $t \in[0,1]$.

Proof of Proposition 3.3. In view of Proposition 2.3 let $u_{1}$ and $u_{0}$ be, respectively, a solution to (3.1.1) and a solution to (3.1.0). Though the uniqueness does not hold, we fix these solutions and proceed further. Put $M=\min _{\bar{\Omega}}\left(u_{1}-u_{0}\right)$ and $N=\max _{\bar{\Omega}}\left(u_{1}-u_{0}\right)$. Then we get

$$
u_{0}+M \leq u_{1} \leq u_{0}+N \text { in } \bar{\Omega}
$$

Note that $u_{0}+M$ and $u_{0}+N$ are also solutions to (3.1.0). Put $\bar{w}=t u_{1}+$ $(1-t)\left(u_{0}+N\right)$ and $\underline{w}=t u_{1}+(1-t)\left(u_{0}+M\right)$. It follows from (3.4) that $\underline{w} \leq u_{1} \leq \bar{w}$ in $\bar{\Omega}$. Here we see that $\underline{w}$ and $\bar{w}$ are, respectively, sub- and supersolutions to (3.1.t) with $\underline{w} \leq \bar{w}$ in $\Omega$. Indeed, since $f$ is nondecreasing, we get

and

$$
\Delta \underline{w}=t f\left(u_{1}\right)+(1-t) k \geq t f(\underline{w})+(1-t) k \quad \text { in } \Omega,
$$

$$
\Delta \bar{w}=t f\left(u_{1}\right)+(1-t) k \leq t f(\bar{w})+(1-t) k \quad \text { in } \Omega .
$$

Of course, $\partial \bar{w} / \partial \nu=c$ and $\partial \underline{w} / \partial \nu=c$ on $\partial \Omega$. Therefore, using the method of sub- and supersolutions (see Sattinger [18, Theorem 2.1, p. 980]), we obtain a solution $u_{t} \in C^{\infty}(\bar{\Omega})$ to (3.1.t) satisfying $\underline{w} \leq u_{t} \leq \bar{w}$ in $\Omega$. Furthermore, in view of (3.4) and the definition of $\underline{w}$ and $\bar{w}$, we get $u_{0}+M \leq u_{t} \leq u_{0}+N$ in $\bar{\Omega}$. Then it follows that

$$
\sup _{\bar{\Omega}}\left|u_{t}\right| \leq C \text { for all } t \in[0,1],
$$

where $C$ is a constant independent of $t \in[0,1]$. So, by the $L_{p}$-estimates of Agmon, Douglis and Nirenberg [1] we see that $\left\|u_{t}\right\|_{W^{2, p}(\Omega)}$ is uniformly bounded with respect to $t \in[0,1]$. Hence, using Sobolev imbedding theorems and the Schauder estimates of [1], we obtain the inequality (3.3). This completes the proof.

Proof of Proposition 3.4. In view of Proposition 2.2, let $u_{1}$ be a unique solution to (3.2.1). Then, since $f$ is nondecreasing, we see that $u_{1}$ is a subsolution to (3.2.t). On the other hand, we observe from Proposition 2.1 that $u_{1} \leq 0$ in $\Omega$ and $f(m)$ is positive. Thus $u \equiv 0$ is a supersolution to (3.2.t), since $f(0)$ is positive. Therefore, using the method of sub- and supersolutions, we obtain a unique solution $u_{t} \in C^{\infty}(\bar{\Omega})$ to $(3.2 . t)$ satisfying $u_{1} \leq u_{t} \leq 0$ in $\Omega$. Hence, 
by the same argument as in the proof of Proposition 3.3 we get the inequality (3.3) and complete the proof.

\section{ONE MOdification OF CHEN AND HuANG'S COMPARISON TECHNIQUE}

We begin with

Lemma 4.1. For any $h$ with $f(h)>0$, there exists a number $L \quad(0<L \leq \infty)$ such that the initial value problem

$$
\left\{\begin{array}{l}
v^{\prime \prime}(s)=f(v(s)) \quad(-L<s<L), \\
v(0)=h \quad \text { and } \quad v^{\prime}(0)=0,
\end{array}\right.
$$

has a unique $C^{\infty}$-solution $v$, which satisfies the following:

$$
\begin{gathered}
v(s)=v(-s) \quad(-L<s<L), \\
v(s) \geq h \quad \text { and } \quad v^{\prime \prime}(s) \geq f(h)>0 \quad(-L<s<L), \\
v^{\prime}(s) \geq 0 \quad(0 \leq s<L), \\
\lim _{s \rightarrow L} v(s)=+\infty \quad \text { and } \lim _{s \rightarrow L} v^{\prime}(s)=+\infty .
\end{gathered}
$$

Proof. The existence and uniqueness theorem for initial value problems for ordinary differential equations implies that there exists a unique $C^{\infty}$-solution $v(s)$ to $(4.1)$ for some positive number $L$. Since $v(-s)$ is also a solution to (4.1), by the uniqueness theorem for initial value problems we get (4.2). Thus, let $(-L, L)$ be a maximal interval of existence for $v$. Put $w(s)=v(s)-h$. Then $w$ satisfies

$$
\left\{\begin{array}{l}
w^{\prime \prime}(s)=f(v(s)) \text { for }-L<s<L, \\
w(0)=w^{\prime}(0)=0 .
\end{array}\right.
$$

Since $w^{\prime \prime}(0)=f(h)>0$, there exists a positive number $\eta$ satisfying $w(s)>0$ for $0<s<\eta$. Here we see that $w(s)>0$ for $0<s<L$. Indeed, suppose that $w\left(s_{0}\right) \leq 0$ for some $s_{0} \geq \eta$. Then we have $\max _{0<s<s_{0}} w(s)=w\left(s_{1}\right)>0$ for some $0<s_{1}<s_{0}$. Hence $w^{\prime \prime}\left(s_{1}\right) \leq 0$. On the other hand, since $w\left(s_{1}\right)$ is positive and $f$ is nondecreasing, $f\left(v\left(s_{1}\right)\right) \geq f(h)>0$. This contradicts the fact that $w^{\prime \prime}\left(s_{1}\right)=f\left(v\left(s_{1}\right)\right)$. Thus, by (4.2) we get

$$
v(s) \geq h \text { for }-L<s<L
$$

and get the whole of (4.3). Furthermore, (4.4) follows from (4.3) and the fact that $v^{\prime}(0)=0$.

When $L=+\infty$, by (4.3) we have for $s>0$

$$
v^{\prime}(s)=\int_{0}^{s} v^{\prime \prime}(t) d t \geq f(h) s
$$

and

$$
v(s)=v(0)+\int_{0}^{s} v^{\prime}(t) d t \geq h+\frac{1}{2} f(h) s^{2} .
$$


Therefore, since $f(h)$ is positive, we get $(4.5)$. When $L$ is finite, since $(-L, L)$ is a maximal interval, we have from (4.3) and (4.4)

$$
\lim _{s \rightarrow L} v(s)=+\infty \text { or } \lim _{s \rightarrow L} v^{\prime}(s)=+\infty .
$$

If $\lim _{s \rightarrow L} v(s)$ is finite, we get for $0<s<L$

$$
v^{\prime}(s)=\int_{0}^{s} f(v(t)) d t \leq f(\sup v) L .
$$

This contradicts $(4.6)$. Therefore we get

$$
\lim _{s \rightarrow L} v(s)=+\infty \text {. }
$$

If $\lim _{s \rightarrow L} v^{\prime}(s)$ is finite, we get, for $0<s<L$,

$$
v(s)=v(0)+\int_{0}^{s} v^{\prime}(t) d t \leq\left(\sup v^{\prime}\right) L+h .
$$

This contradicts (4.7). Thus we get

$$
\lim _{s \rightarrow L} v^{\prime}(s)=+\infty
$$

and complete the proof.

Using one modification of Chen and Huang's comparison technique, we obtain

Lemma 4.2. Let $u \in C^{\infty}(\bar{\Omega})$ be the solution to (3.1.t) or (3.2.t) for $t \in[0,1]$. Suppose that $\nabla u(p)=0$ at some point $p \in \Omega$. Then the Gaussian curvature $K(p)$ of the graph $(x, u(x))$ at $p$ does not vanish, where $K(p)=D_{11} u(p)$. $D_{22} u(p)-\left\{D_{12} u(p)\right\}^{2}$ and $D_{i j}=\partial^{2} / \partial x_{i} \partial x_{j}$.

Proof. As in Remark 3.2, it suffices to show this lemma when $t=1$. Therefore, let $u$ be the solution to (1.5) or (1.6). Let $p \in \Omega$ be a point with $\nabla u(p)=0$. Suppose that $K(p)=0$. Then, by using a parallel translation and a rotation of coordinates, we may assume that

$$
p=0 \quad \text { and } \quad\left[D_{i j} u(0)\right]=\operatorname{diag}[f(u(0)), 0] \text {. }
$$

It follows from Proposition 2.1 that $f(u(0))$ is positive. Using Lemma 4.1 for $h=u(0)$, we get a unique solution to (4.1), say $v$. Put $w(x) \quad\left(=w\left(x_{1}, x_{2}\right)\right)=$ $v\left(x_{1}\right)$. Then $w$ satisfies

$$
\left\{\begin{array}{l}
\Delta w=f(w) \text { in }(-L, L) \times \mathbf{R}, \\
{\left[D_{i j} w(0)\right]=\operatorname{diag}[f(u(0)), 0],} \\
w(0)=u(0) \text { and } \nabla w(0)=\nabla u(0)=0
\end{array}\right.
$$

Hence $u-w$ satisfies

$$
\Delta(u-w)=a(x)(u-w) \text { and } a(x) \geq 0 \quad \text { in }(-L, L) \times \mathbf{R} \cap \Omega,
$$

where $a(x)=\int_{0}^{1} f^{\prime}(w+\theta(u-w)) d \theta$, and $u-w$ vanishes up to second-order derivatives at 0 . Furthermore, since $u-w$ is not identically zero, it follows 
from (4.10) and a unique continuation theorem for solutions to elliptic partial differential equations (see Aronszajn [2]) that $u-w$ never vanishes up to infinite order at 0 . Therefore, by Taylor's formula we get for some integer $n \geq 3$

$$
(u-w)(x)=P_{n}(x)+o\left(|x|^{n}\right) \quad \text { as }|x| \rightarrow 0,
$$

where $P_{n}(x)$ is a homogeneous polynomial of degree $n$ and $P_{n}(x)$ is not identically zero. Furthermore, since $u-w$ is a $C^{\infty}$-function, using (4.10), we see that $P_{n}(x)$ is a harmonic polynomial. On the other hand, it follows from the result of Hartman and Wintner [8, Corollary 1, p. 450] that every interior critical point of $u-w$ is isolated. Therefore, as in [5] we see that the zero set of $u-w$ in some neighborhood $U$ of the origin consists of $n$ smooth arcs, all intersecting at origin and dividing $U$ into $2 n$ sectors $(n \geq 3)$. Put

$$
\begin{aligned}
& A=\{x \in \Omega \cap(-L, L) \times \mathbf{R} ; u(x)-w(x)>0\}, \\
& B=\{x \in \Omega \cap(-L, L) \times \mathbf{R} ; u(x)-w(x)<0\} .
\end{aligned}
$$

Then, it follows from the maximum principle that

Both $A$ and $B$ have at least three components each of which meets the boundary $\partial(\Omega \cap(-L, L) \times \mathbf{R})$.

Now, we first consider the case of Neumann boundary condition (1.5). Furthermore we divide the proof into two cases. One is the case that $L$ is finite, and the other is that $L$ is infinite. Consider the former. Choose a number $L^{\sim}$ with $L^{\sim}<L$, which is sufficiently near to $L$. Put $\Omega^{\sim}=\left(-L^{\sim}, L^{\sim}\right) \times \mathbf{R}$. Look at the boundary $\partial\left(\Omega \cap \Omega^{\sim}\right)$. Since $\Omega$ is convex, observing the boundary condition of $u$ and the shape of the graph of $w$ (see Lemma 4.1), we see that $\partial\left(\Omega \cap \Omega^{\sim}\right)$ consists of at most four connected arcs, in which $\partial(u-w) / \partial \nu$ changes sign alternatively. Put

$$
\begin{aligned}
& \Gamma_{+}=\left\{x \in \partial\left(\Omega \cap \Omega^{\sim}\right) ; \frac{\partial}{\partial \nu}(u-w)(x)>0\right\}, \\
& \Gamma_{-}=\left\{x \in \partial\left(\Omega \cap \Omega^{\sim}\right) ; \frac{\partial}{\partial \nu}(u-w)(x)<0\right\} .
\end{aligned}
$$

(At a corner, we choose $\nu$ to be the unit outer normal vector to $\partial \Omega^{\sim}$.) Then, it never occurs that a component of $A \cap \Omega^{\sim}$ meets $\partial\left(\Omega \cap \Omega^{\sim}\right)$ exclusively in $\Gamma_{-}$. Indeed, let $\omega$ be a component of $A \cap \Omega^{\sim}$ which meets $\partial\left(\Omega \cap \Omega^{\sim}\right)$ exclusively in $\Gamma_{-}$. Hence the strong maximum principle implies that a positive maximum of $u-w$ in $\bar{\omega}$ is attained at $p \in \Gamma_{-}$and $\frac{\partial}{\partial \nu}(u-w)(p) \geq 0$. This contradicts the definition of $\Gamma_{-}$. Also, by the same argument as this, we see that it never occurs that a component of $B \cap \Omega^{\sim}$ meets $\partial\left(\Omega \cap \Omega^{\sim}\right)$ exclusively in $\Gamma_{+}$. However, these facts contradict (4.12).

Next consider the latter when $L$ is infinite. Only replacing $\Omega \cap \Omega^{\sim}$ by $\Omega$, we can use the same argument as above.

In the case of the third kind boundary condition (1.6), replacing $\frac{\partial}{\partial \nu}(u-w)$ by $\frac{\partial}{\partial \nu}(u-w)+\beta(u-w)$, we can use an argument similar to that in the case of 
Neumann boundary condition (1.5). Precisely, we choose $L^{\sim}$ to get $\{w(x)=$ $0\}=\left\{x_{1}= \pm L^{\sim}\right\}$. Then $\partial\left(\Omega \cap \Omega^{\sim}\right)$ consists of at most two components of $\left\{\frac{\partial}{\partial \nu}(u-w)+\beta(u-w)>0\right\}$ and two components of

$$
\{w>0>u\} \cup\left\{\frac{\partial}{\partial \nu}(u-w)+\beta(u-w)<0\right\} .
$$

This completes the proof of Lemma 4.2.

Lemma 4.3. Let $u \in C^{\infty}(\bar{\Omega})$ be the solution to (3.1.0) or (3.2.0). Then $u$ has only one critical point in $\Omega$.

Since we use a continuity argument of $\S 5$ in this paper, we prove this lemma in the last of $\S 5$.

Lemma 4.4. Let $u \in C^{\infty}(\bar{\Omega})$ be the solution to (3.1.t) or (3.2.t). Then $u$ does not have maximal points in $\Omega$.

Proof. Since $\Delta u$ is positive in $\Omega$ (see Remark 3.2 and Proposition 2.1), the strong maximum principle implies this lemma.

Lemma 4.5. Let $u \in C^{\infty}(\bar{\Omega})$ be the solution to $(3.1 . t)$ or $(3.2 . t)$ for $t \in[0,1]$. Then $u$ has more than two minimal points, if and only if there exists a point $p \in \Omega$ with $\nabla u(p)=0$ and $K(p)<0$.

Proof. By Remark 3.2 and Proposition 2.1, we have

$$
\frac{\partial u}{\partial \nu}>0 \text { on } \partial \Omega
$$

Therefore $u$ does not have minimal point on the boundary $\partial \Omega$. We first prove "if" part. Let $p$ be a point with $\nabla u(p)=0$ and $K(p)<0$. Then there exists an open neighborhood $U$ of $p$ in which the zero set of $u-u(p)$ consists of two smooth arcs intersecting at $p$ and divides $U$ into four sectors. Consider the open set $E=\{x \in \Omega ; u(x)>u(p)\}$. It follows from Lemma 4.4 that each component of $E$ has to meet the boundary $\partial \Omega$. Accordingly we see that the open set $G=\{x \in \Omega ; u(x)<u(p)\}$ has more than two components. This shows that $u$ has more than two minimal points.

Next we prove "only if" part. Since $\partial u / \partial \nu$ is positive on $\partial \Omega$ and $\Omega$ is convex, we can extend the function $u$ to $\mathbf{R}^{2}$ by putting, for $x \in \mathbf{R}^{2}-\Omega$,

$$
u(x)=u(y)+\frac{\partial u}{\partial \nu}(y) \times \operatorname{dist}(x, y)
$$

where $y$ is a unique point on $\partial \Omega$ with $\operatorname{dist}(x, y)=\operatorname{dist}(x, \Omega)$. Then we see that $u$ belongs to $C^{1}\left(\mathbf{R}^{2}\right)$ and $\nabla u$ does not vanish in $\mathbf{R}^{2}-\Omega$. Consider the level set $L_{s}=\left\{x \in \mathbf{R}^{2} ; u(x)<s\right\}$. Then it follows that

$$
\partial L_{s} \text { has only one component for sufficiently large } s \text {. }
$$

Suppose that $u$ has more than two minimal points and there exists no point $p$ with $\nabla u(p)=0$ and $K(p)<0$. Therefore, by virtue of Lemma 4.2 and Lemma 4.4 , we see that each critical point of $u$ is a minimal point. Since $\nabla u$ does not vanish in $\mathbf{R}^{2}-\Omega$, Lemma 4.2 implies that every critical point is isolated 
and the number of critical points is finite. Hence we conclude that there exists a finite set of minimal points of $u$, say $\left\{p_{1}, p_{2}, \ldots, p_{N}\right\}$ satisfying

$$
\nabla u(x) \neq 0 \text { for all } x \in \mathbf{R}^{2}-\left\{p_{1}, p_{2}, \ldots, p_{N}\right\} \text {. }
$$

Put $s_{0}=\max \left\{u\left(p_{j}\right) ; 1 \leq j \leq N\right\}$. It follows that the boundary $\partial L_{s}$ of the level set $L_{s}$ is a $C^{1}$-curve for $s>s_{0}$ and $\left\{\partial L_{s}\right\}$ are diffeomorphic to each other. Since $K\left(p_{j}\right)$ is positive, if $s$ is near to $s_{0}, \partial L_{s}$ has more than two components. This contradicts the fact (4.15). This completes the proof.

\section{Proof of Theorem 1 AND Theorem 2}

In view of Lemma 4.2, Lemma 4.4, and Lemma 4.5, it suffices to show that the set of minimal points of the solution consists of one point. Let $u_{t} \in C^{\infty}(\bar{\Omega})$ be the solution to $(3.1 . t)$ or $(3.2 . t)$ for $t \in[0,1]$ obtained by Proposition 3.3 or by Proposition 3.4. Put $I=[0,1]$. Divide $I$ into two sets $I_{1}$ and $I_{2}$ : $I_{1}=\left\{t \in I ; u_{t}\right.$ has only one minimal point $\}, I_{2}=\left\{t \in I ; u_{t}\right.$ has more than two minimal points $\}$. Then $I=I_{1} \cup I_{2}$ and $I_{1} \cap I_{2}=\varnothing$. It follows from Lemma 4.3 that 0 belongs to $I_{1}$, namely, $I_{1}$ is not empty. Furthermore, Lemma 4.2 and the inequality (3.3) imply that $I_{2}$ is open in $I$. (See Proposition 3.3 and Proposition 3.4. Though the uniqueness of the solution to (3.1.t) does not hold, since the solution to (3.1.t) is unique up to an additive constant, so $t \in I_{2}$ if and only if there exists a solution to (3.1.t) which has more than two minimal points.) Thus it remains to show that $I_{2}$ is closed in $I$. Let $\left\{t_{j}\right\}$ be a sequence of points in $I_{2}$ such that $t_{j}$ converges to $t_{*}$ as $j$ tends to $\infty$. Hence, Lemma 4.5 and the compactness arguments imply that there exist a subsequence $\left\{t_{k}\right\}$, a sequence of points $\left\{p_{k}\right\}$, and a point $p \in \bar{\Omega}$ which satisfy

$$
p_{k} \rightarrow p \quad \text { as } k \rightarrow \infty, \quad \nabla u_{t_{k}}\left(p_{k}\right)=0, \quad \text { and } \quad K_{t_{k}}\left(p_{k}\right)<0
$$

By continuity we have

$$
\nabla u_{t_{*}}(p)=0 \text { and } K_{t_{*}}(p) \leq 0 .
$$

Here, in the case of (3.1.t) we used the fact that the solution is unique up to an additive constant. Since $\nabla u_{t_{*}} \neq 0$ on $\partial \Omega$, we have $p \in \Omega$. Therefore it follows from Lemma 4.2, Lemma 4.5, and (5.2) that $t_{*}$ belongs to $I_{2}$. This shows that $I_{2}$ is closed in $I$. The proof is now completed.

Proof of Lemma 4.3. Since the equations in (3.1.0) and (3.2.0) are linear, by introducing the smooth deformation of the domain $\Omega$ into a ball as in [3] or [14] and by using the continuity argument as above, we prove this lemma. Precisely, let $\Omega_{s} \quad(0 \leq s \leq 1)$ be a family of bounded convex smooth domains such that $\Omega_{0}=$ a ball, $\Omega_{1}=\Omega$ and $\partial \Omega_{s}$ varies smoothly in the parameter $s$. Consider the following two families of linear problems:

$$
\left\{\begin{array}{l}
\Delta v=k \text { in } \Omega_{s}, \\
\partial v / \partial \nu=c_{s} \text { on } \partial \Omega_{s}, \\
\int_{\Omega_{s}} v d x=0
\end{array}\right.
$$


where $c_{s}=\left|\Omega_{s}\right| k /\left|\partial \Omega_{s}\right|$, and

$$
\left\{\begin{array}{l}
\Delta v=f(m) \text { in } \Omega_{s}, \\
\partial v / \partial \nu+\beta v=0 \text { on } \partial \Omega_{s} .
\end{array}\right.
$$

Let $v_{s}$ be a unique solution to $(5.3 . s)$ or $(5.4 . s)$. Then it follows from the Schauder global estimates that $C^{2+\alpha}$-norm of $v_{s}$ is uniformly bounded with respect to $s$. Since $v_{0}$ is radial, $v_{0}$ has only one critical point in $\Omega_{0}$. Therefore, using Lemma 4.2, Lemma 4.4, Lemma 4.5 and the continuity argument of this section, we see that $v_{1}$ has only one critical point in $\Omega\left(=\Omega_{1}\right)$. This completes the proof.

Acknowledgments. I would like to thank Professor H. Matano for informing me of the papers Payne [17] and Sperb [19]. Also, I am grateful to Professor M. Murata for telling me the use of a unique continuation theorem due to Aronszajn [2].

\section{REFERENCES}

1. S. Agmon, A. Douglis, and L. Nirenberg, Estimates near the boundary for solutions of elliptic partial differential equations satisfying general boundary conditions. I, Comm. Pure Appl. Math. 12 (1959), 623-727.

2. N. Aronszajn, A unique continuation theorem for solutions of elliptic partial differential equations or inequalities of second order, J. Math. Pures Appl. 36 (1957), 235-249.

3. L. A. Caffarelli and A. Friedman, Convexity of solutions of semilinear elliptic equations, Duke Math. J. 52 (1985), 431-456.

4. L. A. Caffarelli and J. Spruck, Convexity properties of solutions to some classical variational problems, Comm. Partial Differential Equations 7 (1982), 1337-1379.

5. Jin-Tzu Chen and Wu-Hsiung Huang, Convexity of capillary surfaces in the outer space, Invent. Math. 67 (1982), 253-259.

6. Jin-Tzu Chen, Uniqueness of minimal point and its location of capillary free surfaces over convex domain, Astérisque, no. 118, Soc. Math. France, Paris, 1984, pp. 137-143.

7. D. Gilbarg and N. S. Trudinger, Elliptic partial differential equations of second order, Springer-Verlag, 1977.

8. P. Hartman and A. Wintner, On the local behavior of solutions of non-parabolic partial differential equations, Amer. J. Math. 75 (1953), 449-476.

9. B. Kawohl, When are solutions to nonlinear elliptic boundary value problems convex?, Comm. Partial Differential Equations 10 (1985), 1213-1225.

10. __ Rearrangements and convexity of level sets in PDE, Lecture Notes in Math., vol. 1150, Springer-Verlag, 1985.

11. A. U. Kennington, Power concavity and boundary value problems, Indiana Univ. Math. J. 34 (1985), 687-704.

12. N. Korevaar, Capillary surface convexity above convex domains, Indiana Univ. Math. J. 32 (1983), 73-81.

13. Convex solutions to nonlinear elliptic and parabolic boundary value problems, Indiana Univ. Math. J. 32 (1983), 603-614.

14. N. Korevaar and J. L. Lewis, Convex solutions of certain elliptic equations have constant rank Hessians, Arch. Rational Mech. Anal. 97 (1987), 19-32.

15. G. M. Lieberman, The conormal derivative problem for elliptic equations of variational type, J. Differential Equations 49 (1983), 218-257. 
16. G. M. Lieberman and N. S. Trudinger, Nonlinear oblique boundary value problems for nonlinear elliptic equations, Trans. Amer. Math. Soc. 295 (1986), 509-546.

17. L. E. Payne, On two conjectures in the fixed membrane eigenvalue problem, $\mathrm{Z}$. Angew. Math. Phys. 24 (1973), 721-729.

18. D. H. Sattinger, Monotone methods in nonlinear elliptic and parabolic boundary value problems, Indiana Univ. Math. J. 21 (1972), 979-1000.

19. R. P. Sperb, Extension of two theorems of Payne to some nonlinear Dirichlet problems, Z. Angew. Math. Phys. 26 (1975), 721-726.

Numazu College of Technology, 3600 Oooka, Numazu-shi, Shizuoka 410, Japan

Current address: Department of Mathematics, Tokyo Institute of Technology, Oh-Okayama, Meguro, Tokyo 152, Japan 